TRANSACTIONS OF THE

AMERICAN MATHEMATICAL SOCIETY

Volume 358, Number 10, October 2006, Pages 4521-4531

S 0002-9947(06)03998-5

Article electronically published on March 24, 2006

\title{
GENERIC SYSTEMS OF CO-RANK ONE VECTOR DISTRIBUTIONS
}

\author{
HOWARD JACOBOWITZ
}

\begin{abstract}
This paper studies a generic class of sub-bundles of the complexified tangent bundle. Involutive, generic structures always exist and have Levi forms with only simple zeroes. For a compact, orientable three-manifold the Chern class of the sub-bundle is mod 2 equivalent to the Poincare dual of the characteristic set of the associated system of linear partial differential equations.
\end{abstract}

Let $M^{n}$ be a compact manifold. We study generic sub-bundles of co-rank one. In defining a condition of genericity, we are guided by the needs of the theory of linear partial differential equations and by the theory of generic functions as initiated by Whitney. We use $V$ to denote a sub-bundle of $C T M$ of co-rank one. Such a sub-bundle defines a map $\Phi_{V}: M \hookrightarrow P C T^{*} M$, where the image of a point $p$ is the line of one-forms which annihilate $V_{p}$. We are interested in points where this line is generated by a real form, so we set

$$
\Sigma(V)=\left\{p \in M: \Phi_{V}(p) \in P T^{*} M\right\} .
$$

This is the characteristic set of the system of first order partial differential operators corresponding to any local basis for $V$. In particular, the system is elliptic outside of $\Sigma$. As we have just done, we freely write $\Sigma$ for $\Sigma(V)$. All manifolds, bundles, and maps are taken to be of class $C^{\infty}$.

Definition 1. $V$ is an almost Whitney structure if

(1) $\Phi_{V}(M)$ intersects $P T^{*} M$ transversely. It follows that $\Sigma$ is a smooth curve with a finite number of components.

(2) $\omega(\tau)$ has only simple zeroes when $\tau$ is a nonzero section of $T \Sigma$ and $\omega$ is a one form such that $\Phi_{V}=[\omega]$ on $\Sigma$.

Remark 0.1. We follow the convention of introducing "almost" so that we can drop it once we require formal integrability, i.e. involutivity. Thus $V$ is a Whitney structure if in addition

(3) $[V, V] \subset V \cdot($ See $\S 3$.

Example. Let $M=X \times S^{1}$, where $X$ is a compact two-dimensional manifold (or, more generally, let $M$ be a circle bundle over $X$ with orientable fiber). Let $\xi$ be a real nondegenerate vector field on $X$ with $\{p \in X: \xi(p)=0\}=\left\{p_{1}, \ldots, p_{N}\right\}$. That is, at each $p_{j}$ the linear approximation to $\xi$ is given by a matrix whose determinant is

Received by the editors September 8, 2004.

2000 Mathematics Subject Classification. Primary 58J10; Secondary 57R20.

Key words and phrases. Generic sub-bundle, Chern class, Levi form.

(C)2006 American Mathematical Society 
nonzero. This is equivalent to requiring that the section $\xi: M \rightarrow T M$ is transverse to the zero section. Use some metric to identify the vector field $\xi$ with a one-form $\omega_{0}$ and set $\omega=\omega_{0}-i d \theta$, where $\theta$ is the natural coordinate on $S^{1}$. Let

$$
V=\{v \in C T M: \omega(v)=0\} .
$$

For $\xi=\xi_{1} \partial_{x}+\xi_{2} \partial_{y}$ near $p_{j}$ and with an appropriate choice of metric near $p_{j}$, we have $\omega=\xi_{1} d x+\xi_{2} d y-i d \theta$ and local sections for $V$

$$
\begin{aligned}
& v_{1}=\partial_{x}-i \xi_{1} \partial_{\theta}, \\
& v_{2}=\partial_{y}-i \xi_{2} \partial_{\theta} .
\end{aligned}
$$

Note that

$$
\Sigma=\bigcup\left\{p_{j} \times S^{1}\right\}
$$

Further, $\Phi_{V}(M)$ intersects $P T^{*}(M)$ transversely because $\xi$ is nondegenerate. For $\tau$ we take $\partial_{\theta}$, so $\omega(\tau)$ is never zero. Thus $V$ is an almost Whitney structure. We return to this example in the next section.

Theorem 0.1. The almost Whitney structures are generic.

Proof. By generic, we mean that the set of Whitney structures is open in the $C^{1}$ topology (this is clear) and that any $V$ may be approximated in the $C^{\infty}$ topology by a Whitney structure. Any $C^{\infty} V$ determines a $C^{\infty}$ map $\Phi_{V}: M \rightarrow P C T^{*} M$. By a standard result of differential topology, $\Phi_{V}$ may be perturbed, within the class of $C^{\infty}$ maps, to obtain a map $\tilde{\Phi}$ with $\tilde{\Phi}(M)$ transverse to $P T^{*} M$. To $\tilde{\Phi}$ there corresponds some $\tilde{V}$. Thus we may assume that $V$ itself satisfies the transversality condition (1) and turn our attention to condition (2). We have that $\Sigma=\{p \in M$ : $\Re \omega \wedge \Im \omega=0\}$. We seek a perturbation $\tilde{\omega}$ which agrees with $\omega$ away from $\Sigma$ and for which $\tilde{\omega}(\tau)$ has only simple zeroes. So let $\theta$ be a parametrization of $\Sigma, r$ a smooth function with $\Sigma=\{p \in M: r(p)=0\}$ and $a(r, \theta)$ some small real-valued function which is zero on the complement of some small neighborhood of $\Sigma$. Set

$$
\tilde{\omega}=\omega+a(r, \theta) d \theta \text {. }
$$

Then on $\Sigma$

$$
\tilde{\omega}(\tau)=\omega(\tau)+a(r, \theta) d \theta(\tau)
$$

and for a generic choice of $a(0, \theta), \tilde{\omega}(\tau)$ has only simple zeroes.

\section{TOpological CONSEQUences}

When involutivity is ignored, the study of co-rank one bundles is equivalent to the study of rank one bundles. (This is made precise in Lemma[1.1) A complex line bundle $Q \subset C T M$ with $\Sigma$ empty is the same as an oriented two-plane distribution in $T M$. Here are some known results for compact, orientable manifolds which admit oriented two-plane distributions in $T M$. There seems to be little in the literature about similar questions for nonorientable manifolds or distributions.

If $\operatorname{dim} M=4 k: \chi(M)$ is even and is congruent to the signature of $M$ modulo 4.

If $\operatorname{dim} M=4 k+1: k(M)=0$.

If $\operatorname{dim} M=4 k+2$ : The first Chern class of the associated complex line bundle satisfies

$$
2\left(c_{1} \wedge v\right)[M]=\chi(M)
$$

for some $v \in H^{4 k-2}(M, Z)$ with $v \bmod 2=w_{4 k-2}(M)$. 
If $\operatorname{dim} M=4 k+3:$ The first Chern class satisfies

$$
\sum_{2 l+j=4 k+2} c_{1}^{l} \wedge w_{j}(M)=0
$$

as an element of $H^{4 k+2}\left(M, Z_{2}\right)$.

Here $\chi(M)$ is the Euler characteristic, $k(M)$ is the Kervaire semi-characteristic, and $w_{j}(M)$ is the Steifel-Whitney class of $T M$. The first two results are due to Atiyah [1] and the others to Thomas [7] and [8]. Note that the converses of the latter also hold, op cit. For a three-dimensional manifold, Thomas' result says that if $V \subset C T M$ has $\Sigma$ empty, then $c_{1}(V)$ is zero as an element of $H^{2}\left(M, Z_{2}\right)$. It follows that $c_{1}(V)$ is even in the sense that there exists some $\alpha \in H^{2}(M, Z)$ with $c_{1}(V)=2 \alpha$.

In general, Thomas studies the existence of a two-plane distribution on $M$ by using the skeleton of $M$ to show that there always exists a two-plane distribution on $M-\{$ finite set of points $\}$ and then finding the obstruction to extending to all of $M$ (see [8], page 350). The present work suggests a different approach to a related problem. A generic embedding of a complex line bundle into $C T M^{n}$ produces a two-plane distribution on $M-\Sigma$, where $\Sigma$ is a union of circles. One can then try to relate the Chern class of the bundle to a multiple of the homology class $[\Sigma]$. Instead of studying two-plane distributions on $M-\{$ finite set of points\}, this approach studies two-plane distributions on $M-$ finite union of disjoint circles\} which only degenerate to line elements of $M$.

In dimension two, there are precise results.

Theorem 1.1 ([3]). If $V$ is a generic structure on a compact, orientable $M^{2}$, then

$$
\text { degree }(V)=\chi\left(M^{+}\right)-\chi\left(M^{-}\right)-\# \text { cusps. }
$$

For this theorem, an orientation of $M$ is fixed. Then, off of $\Sigma, V$ defines an orientation for $M$ which agrees with the chosen one for points of $M^{+}$. The degree of $V$ is $c_{1}(V)$ evaluated on the basic homology class $[M]$, and the number of cusps is the number of times that $V$ contains the tangent space to $\Sigma$.

We now briefly return to the general dimension. We have that on $\Sigma, V=C \otimes W$, where $W$ is a real bundle of rank $n-1$.

Definition 2. $\Sigma(V)$ is regular if $W$ is transversely orientable.

This means that a direction transverse to $W$ can be consistently chosen. When $M$ is orientable, this is the same as requiring that $W$ is orientable along $\Sigma$.

The purpose of this section is to prove the following new result for $\operatorname{dim} M=3$.

Theorem 1.2. If $V$ is a generic structure on a compact, orientable $M^{3}$ and $\Sigma$ is regular, then

$$
c_{1}(V) \equiv P D[\Sigma] \bmod 2 .
$$

Remark 1.1. A similar formula is probably true without the restriction that $\Sigma$ is regular.

We are using the Poincaré dual of the homology class defined by $\Sigma$. The orientability of $M$ is only used at the last step of the proof. Thus, until then, when $M$ is nonorientable, $P D[\Sigma]$ is the mod 2 Poincaré dual and is an element of $H^{2}\left(M, Z_{2}\right)$. The theorem means that there exists a second cohomology class $\alpha \in H^{2}(M, Z)$ with $c_{1}(V)-P D[\Sigma]=2 \alpha$. 
Example (continued). The bundle $V$ defined in (1) has a trivial bundle as its annihilator. Since the tangent bundle of a compact, orientable three manifold is trivial, it follows that $c_{1}(V)$ is zero (and that $V$ is trivial). Thus the left-hand side of (2) is zero. Since $\Sigma=\bigcup\left\{p_{j} \times S^{1}\right\}$, we have $P D(\Sigma)=N\left[S^{1}\right]$, where $S^{1}$ is the typical fiber and $N$ is the number of zeroes of the vector field $\xi$. If $X$ is orientable, then $N$ is even, and so the right-hand side of Equation (2) is also equal to zero modulo 2. However, if $X$ is nonorientable, then $N$ can be odd. Thus Theorem 1.2 need not hold when $M$ is nonorientable.

Let $Q$ be any complex line bundle in $C T M$ that is complementary to $V$,

$$
C T M=V \oplus Q .
$$

Again making use of the fact that the tangent bundle of a compact, orientable three manifold is trivial, we may prove the theorem by proving that

$$
c_{1}(Q) \equiv P D[\Sigma] \bmod 2 .
$$

Note that $\Sigma(V)$ is regular if and only if $Q=C \otimes B$ on $\Sigma$, where $B$ is a trivial real line bundle. Let $\Phi_{Q}$ be the associated map of $M$ into PCTM and let $\Sigma(Q)$ be the projection into $M$ of the set $\Phi_{Q}(M) \cap P T M$.

Lemma 1.1. For any $V$ and $Q$ satisfying (3), $\Sigma(Q)=\Sigma(V)$. Further, $\Phi_{V}(M)$ intersects $P T^{*} M$ transversely if and only if $\Phi_{Q}$ intersects PTM transversely.

Any line bundle $Q$ satisfying this transversality condition is called generic. At first, we need only that $\Sigma$ is smooth. Transversality is needed only in Lemma 1.6.

Let $\mathcal{N}$ be a small tubular neighborhood of $\Sigma$. (We might need to replace $\mathcal{N}$ by a smaller tubular neighborhood; if so, we do it without explicitly saying so.) Denote its boundary by $b d y \mathcal{N}$. We now take $\Sigma$ to be regular, and so $Q$ restricted to $\Sigma$ is of the form $C \otimes B$, where $B$ is a trivial real line bundle.

Lemma 1.2. There exist real vector fields $U$ and $T$ on $\mathcal{N}$ such that $U+i T$ is a section of $Q, U \wedge T \neq 0$ on bdy $\mathcal{N}$ and $\Sigma=\{p \in M: T(p)=0\}$.

Proof. Let $U_{0}$ be a nonzero section of $B$. Extend it to be a nonzero section of $T \mathcal{N}$ and find other sections $X_{1}$ and $X_{2}$ such that $U_{0} \wedge X_{1} \wedge X_{2} \neq 0$ on $\mathcal{N}$. Since $\left.Q\right|_{\mathcal{N}}$ is trivial, we can find a nonzero section $\sigma$. We have

$$
\sigma=\alpha U_{0}+\beta_{1} X_{1}+\beta_{2} X_{2} .
$$

$\alpha$ is nowhere zero while $\beta_{1}$ and $\beta_{2}$ are both zero on $\Sigma$. Set

$$
\begin{aligned}
\sigma_{1}=\sigma / \alpha & =U_{0}+\beta_{3} X_{1}+\beta_{4} X_{2} \\
& =U_{0}+a_{1} X_{1}+a_{2} X_{2}+i\left(b_{1} X_{1}+b_{2} X_{2}\right) \\
& =U+i T .
\end{aligned}
$$

Since $U+i T$ is a section of $Q$, it follows that $U \wedge T \neq 0$ off of $\Sigma$. Also, clearly $T=0$ on $\Sigma$. So the lemma is proved.

We now choose a vector field $V$ so that $U \wedge T \wedge V>0$ near $b d y \mathcal{N}$ and we choose a metric on $T \mathcal{N}$ and the orientation on $M$ so that near $b d y \mathcal{N}$ we have that $\{U, T, V\}$ is orthonormal and oriented. We also choose an orthonormal and oriented frame $\{U, X, Y\}$ over $\mathcal{N}$. 
Lemma 1.3. The bundle $Q$ can be deformed globally over $M$ to a bundle $Q_{1}$ with

$$
Q_{1}=\left\{\begin{array}{cc}
Q & \text { away from } b d y \mathcal{N} \\
T+i V & \text { near bdy } \mathcal{N}
\end{array}\right.
$$

Hence $\Sigma\left(Q_{1}\right)=\Sigma(Q)$.

Proof. Let $\lambda$ be some smooth function which is supported near $b d y \mathcal{N}$ and which is identically equal to one on some open set containing $b d y \mathcal{N}$. We may assume that $U+i T$ is defined in a neighborhood of $\mathcal{N}$ and that this neighborhood contains the support of $\lambda$. Define a deformation by

$$
Q_{t}=\left\{\begin{array}{cc}
\operatorname{span}\left\{T+i \cos \left(\frac{\pi}{2} t \lambda\right) U+i \sin \left(\frac{\pi}{2} t \lambda\right) V\right\} & \begin{array}{c}
\text { inside the support of } \lambda, \\
\text { outside the support of } \lambda .
\end{array}
\end{array}\right.
$$

$Q_{t}$ is well defined because $\operatorname{span}\{T+i U\}=Q$ near $\mathcal{N}$. Clearly $Q_{1}$ has the desired form.

Let $H=U^{\perp}$. The metric and orientation give $H$ the structure of a complex line bundle with section $X+i Y$. Near $b d y \mathcal{N}$, this structure is also given by $T+i V$. Thus there exists a unique complex-valued function $f: b d y \mathcal{N} \rightarrow S^{1} \subset C-\{0\}$ satisfying

$$
X+i Y=f(p)(T+i V), \quad p \in b d y \mathcal{N} .
$$

Now let $\Sigma_{0}$ be one component of $\Sigma$ and $\mathcal{N}_{0}$ the corresponding component of $\mathcal{N}$. Let $\theta$ parametrize the circle $\Sigma_{0}$ and let $\psi$ parametrize the orthogonal circle in $b d y \mathcal{N}_{0}=T^{2}$. These correspond to coordinates $(\theta, r, \psi)$ for $\mathcal{N}_{0}$, where $(r, \psi)$ are polar coordinates on the planes $\left\{\theta=\theta_{0}\right\}$. Fix some value for $\theta$ and let $m$ be the winding number,

$$
m=\frac{1}{2 \pi i} \int_{\theta=\theta_{0}} \frac{d f}{f} .
$$

Let $F: \mathcal{N}_{0} \rightarrow \mathcal{D}$ be a smooth extension of $f$ to a map into the unit disc, chosen so that 0 is a regular value for $F$. Then $F^{-1}(0)$ is the union of circles and $\left[F^{-1}(0)\right]$ is an element of the first homology group $H_{1}(M, Z)$.

Lemma 1.4. $\left[F^{-1}(0)\right] \equiv m\left[\Sigma_{0}\right] \bmod 2$.

This means that there is some $\alpha \in H_{1}(M, Z)$ for which

$$
\left[F^{-1}(0)\right]-m\left[\Sigma_{0}\right]=2 \alpha .
$$

Remark 1.2. Of course, the same result holds for $f: b d y \mathcal{N}_{0} \rightarrow C-(0)$.

Proof. Let $\mathcal{D}$ be the disc $\left\{\left(r, \psi, \theta_{0}\right)\right\} \subset \mathcal{N}_{0}$ transverse to $\Sigma_{0}$. $\mathcal{D}$ has an induced orientation, and we assume that $\psi$ was chosen consistent with this orientation. We may also assume that $F^{-1}(0)$ is transverse to $\mathcal{D}$. Let $f_{0}=\left.F\right|_{\mathcal{D}}$. Each zero of $f_{0}$ may be assigned \pm 1 , depending on whether $f_{0}$ is locally orientation-preserving or reversing there. The algebraic number of zeroes is equal to $m$, and so

$$
m \equiv \#\left\{f_{0}=0\right\} \bmod 2 .
$$

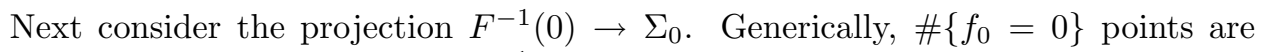
mapped to one point, and so $\left[F^{-1}(0)\right]=m\left[\Sigma_{0}\right] \bmod 2$. 
We now need a general observation about complex line bundles. Let $P_{1}$ and $P_{2}$ be complex line bundles on a compact manifold $M^{3}$. Let $\gamma$ be a smooth curve in $M$ and $\mathcal{N}$ a tubular neighborhood of $\gamma$. Assume that $P_{1}$ and $P_{2}$ are isomorphic over $M-\gamma$ and denote an isomorphism by $\Phi$. Since both $P_{1}$ and $P_{2}$ are trivial over $\mathcal{N}$, they have nonzero sections $\zeta_{1}$ and $\zeta_{2}$. Near $b d y \mathcal{N}$ we have

$$
\Phi \zeta_{1}=f \zeta_{2}
$$

for some $f: b d y \mathcal{N} \rightarrow C-\{0\}$. As before, let $\theta$ parametrize $\gamma$ and set

$$
m=\frac{1}{2 \pi i} \int_{\theta=\theta_{0}} \frac{d f}{f}
$$

Lemma 1.5. $c_{1}\left(P_{2}\right) \equiv c_{1}\left(P_{1}\right)+m P D(\gamma) \bmod 2$.

Proof. Extend $\zeta_{1}$ to a generic global section. Then, as is well known, $c_{1}\left(P_{1}\right)=$ $P D\left[\zeta_{1}^{-1}(0)\right]$. As before let $F$ be a generic extension of $f$ to a map $\mathcal{N} \rightarrow C-\{0\}$. Consider the smooth section $\hat{\zeta}_{2}: M \rightarrow P_{2}$ defined by

$$
\hat{\zeta}_{2}=\left\{\begin{array}{cc}
\Phi \zeta_{1} & \text { on } M-\mathcal{N} \\
F \zeta_{2} & \text { on } \mathcal{N}
\end{array}\right.
$$

Since $\hat{\zeta}_{2}^{-1}(0)=\zeta_{1}^{-1}(0) \cup F^{-1}(0)$, we have

$$
\begin{aligned}
c_{1}\left(P_{2}\right) & =P D\left[\hat{\zeta}_{2}^{-1}(0)\right] \\
& =P D\left[\zeta_{1}^{-1}(0)\right]+P D\left[F^{-1}(0)\right] \\
& \equiv c_{1}\left(P_{1}\right)+m[\gamma] \bmod 2 .
\end{aligned}
$$

We apply this lemma to a complex line bundle $Q \subset C T M^{3}$ with

$$
\Sigma=\bigcup_{1}^{K} \Sigma_{j}
$$

where each $\Sigma_{j}$ is a closed curve. We modify $Q$ in a neighborhood of $\Sigma_{1}$ to obtain a bundle $Q_{1} \subset C T M$ with

$$
\Sigma\left(Q_{1}\right)=\bigcup_{2}^{K} \Sigma_{j}
$$

and

$$
c_{1}(Q) \equiv c_{1}\left(Q_{1}\right)+m_{1} P D\left[\Sigma_{1}\right] \bmod 2 .
$$

Then $Q_{1}$ is modified in a neighborhood of $\Sigma_{2}$ to get

$$
\Sigma\left(Q_{2}\right)=\bigcup_{3}^{K} \Sigma_{j}
$$

and

$$
c_{1}\left(Q_{1}\right) \equiv c_{1}\left(Q_{2}\right)+m_{1} P D\left[\Sigma_{1}\right] \bmod 2 .
$$

We end with $Q_{K} \subset C T M$, where

$$
c_{1}\left(Q_{K-1}\right) \equiv c_{1}\left(Q_{K}\right)+m_{K} P D\left[\Sigma_{K}\right] \bmod 2
$$

and $\Sigma\left(Q_{K}\right)$ is empty. But on an orientable compact three-manifold, this last condition implies that $c_{1}\left(Q_{K}\right) \equiv 0 \bmod 2$. See, for instance the simplest case of Theorem 1.1 of [7] or, for an elementary proof, [5]. Thus

$$
c_{1}(Q) \equiv \sum_{1}^{K} m_{j} P D\left[\Sigma_{j}\right] \bmod 2 .
$$


To evaluate $m_{j}$, we add the assumption that $Q$ is generic.

Lemma 1.6. Let $Q$ be a generic complex line bundle with $\Sigma=\bigcup_{j=1}^{K} \Sigma_{j}$. Then

$$
c_{1}(Q) \equiv P D[\Sigma] \bmod 2 .
$$

Proof. It is enough to work with one component, $\Sigma_{0}$, of $\Sigma$ and show that the associated value of $m$ is \pm 1 . We know that near this component $Q$ has a section $U+i T$ as in Lemma1.2. If necessary, we deform $Q$ near $\Sigma_{0}$ to obtain that $U$ is never tangent to $\Sigma_{0}$. As before, we have that $\{U, T, V\}$ is an orthonormal and oriented basis over a neighborhood of $b d y \mathcal{N}_{0}$ and $\{U, X, Y\}$ is an orthonormal and oriented basis over a neighborhood of $\mathcal{N}_{0}$. We use this latter basis to provide homogeneous coordinates for PCTM near $\Sigma_{0}$. We have $T=a U+b X+c Y$ with $a=b=c=0$ on $\Sigma_{0}$. Then

$$
\begin{aligned}
\pi_{Q}(p) & =(p,[1+i a, i b, i c]) \\
& =(p,[1, B, C]) .
\end{aligned}
$$

Introduce coordinates $(\theta, s, t)$ with $\left(\theta_{0}, s, t\right)$ parametrizing the disc $\mathcal{D}$. Since $b$ and $c$ vanish on $\Sigma_{0}$ while $P T M$ is given by $\Im B=\Im C=0$, the transversality condition is precisely that the determinant of the Jacobian $\frac{\partial(b, c)}{\partial(s, t)}$ not vanish anywhere on $\Sigma_{0}$. Near $b d y \mathcal{N}_{0}$ we have that $a=0$ and so $V=-c X+b Y$ and

$$
X+i Y=f(T+i V)
$$

with $f=(b-i c)^{-1}$.

To evaluate $m$, we use the coordinate transformation $(s, t) \rightarrow \zeta=b-i c$. The sign of the determinant of the Jacobian determines whether this coordinate change preserves or reverses orientation. We have

$$
2 \pi i m=\int \frac{d f}{f}=-\int \frac{d(b-i c)}{b-i c}=-\int \frac{d \zeta}{\zeta}= \pm 2 \pi i .
$$

Note that we did not actually use transversality everywhere along $\Sigma_{0}$ but only at some point $\theta=\theta_{0}$.

\section{INVOLUTIVITY}

The most interesting systems of partial differential equations are involutive. So we now review this condition and show the existence of Whitney structures. Let $W \subset C T M$ have any rank.

Definition 3. $W$ is involutive if $[W, W] \subset W$.

This means that the vector commutator (Lie bracket) of two local sections of $W$ is also a section of $W$.

There are some standard examples of involutive structures, among which are

- Foliations: $W \subset T M$.

- Complex manifolds: $W \oplus \bar{W}=C T M$.

- CR manifolds: $W \cap \bar{W}=\{0\}$.

The involutivity condition is roughly the same as (more precisely, follows from) the existence of the correct number of independent first integrals. We wish to construct an involutive system of co-rank one, so we can start with one global function which 
will be our first integral and then find the system. That is, we start with a solution and then find the equations. All we need is a function

$$
f: M^{n} \rightarrow C
$$

with $d f \neq 0$. Then

$$
V=\{d f\}^{\perp}
$$

satisfies

$$
[V, V] \subset V .
$$

Note that $\Sigma=\{p \in M: d f \wedge d \bar{f}=0$ at $p\}$.

Clearly, such functions exist. For instance, one could use $f=a+i b$, where $a$ and $b$ are a generic choice of Morse functions. So the existence of a co-rank one involutive structure on an arbitrary manifold is not in doubt. But we would like as much control as possible over the characteristic set of the structure. This means choosing $a$ and $b$ so as to satisfy the conditions of an almost Whitney structure. This is done by taking $p \rightarrow(a(p), b(p))$ to be a generic map into $R^{2}$, instead of a pair of generic maps into $R^{1}$. The classical work of Whitney and Levine studies generic maps into $R^{2}$ (and originated in an attempt to generalize Morse theory).

Let $\mathcal{F}(M)$ denote the set of smooth maps $f=(u, v)$ of $M^{n} \rightarrow R^{2}$ with the property that whenever $d u \wedge d v=0$ at $p$, then there are local coordinates near $p$ and near $f(p)$ such that $f$ has one of the forms

(1)

$$
f(x, t)=\left(x, \sum_{j} \pm t_{j}^{2}\right)
$$

or

$$
f(x, y, t)=\left(x, \sum_{j} \pm t_{j}^{2}+x y \pm y^{3}\right) .
$$

Theorem $2.1([6]) . \mathcal{F}(M)$ is dense in $C^{\infty}\left(M, R^{2}\right)$.

We note that when $\operatorname{dim} M=2, \mathcal{F}(M)$ coincides with the set of excellent maps introduced by Whitney [9]. Recall that for

$$
f(x, y)=(u(x, y), v(x, y))
$$

and

$f$ is excellent when

$$
J(x, y)=\operatorname{det}\left|\begin{array}{ll}
u_{x} & u_{y} \\
v_{x} & v_{y}
\end{array}\right|
$$

- $J(p)=0 \Rightarrow d J(p) \neq 0$. So $\Sigma=\{p: J(p)=0\}$ is smooth and rankf $\geq 1$, and

- $\left.f\right|_{\Sigma}$ has only nondegenerate critical points.

Let $f \in \mathcal{F}$, identify $R^{2}$ with $C$ and set $V=\left\{d f^{\perp}\right\}$. Then $V$ is a Whitney structure.

Conjecture 2.1. The Whitney structures are open and dense in the space of all involutive co-rank one sub-bundles of CTM.

It is natural to wonder about involutive structures of higher co-rank. 
Conjecture 2.2. Every manifold admits involutive structures of all ranks up to the dimension.

The conjecture is true for rank greater than half the dimension [4. A well-known vanishing theorem of Bott for real or holomorphic foliations can be restated to show that there are restrictions on the Chern class of an involutive structure. We present a special case of this argument in $\S 4$.

\section{The LeVI FORM}

We introduce the Levi form of $V$ and show that it has nice properties when $V$ is Whitney. This suggests that the analysis of the corresponding system of partial differential equations should be amenable to classical techniques. Thus if Conjecture 2.1 holds, it should be possible to study generic involutive co-rank one systems of linear partial differential operators.

In general, a Levi form $\mathcal{L}(P, Q)$ is defined for any sub-bundle $W$ as

$$
\mathcal{L}(P, Q)=[P, \bar{Q}] \bmod W+\bar{W},
$$

where $P$ and $Q$ are local sections of $W$. Note that $\mathcal{L}(P, Q)$ is a bilinear form on the fibers of $W+\bar{W}$. That is, it depends only on the pointwise values of $P$ and $Q$. Now let $V$ be a co-rank one sub-bundle (so $\mathcal{L}$ is only interesting at the points of $\Sigma$ ) and choose a global nowhere zero section $\omega$ for $V^{\perp}$ in a neighborhood of $\Sigma$. We use the following equivalent definition of this Levi form.

Definition 4. $\mathcal{L}(P, \bar{Q})=\omega[P, \bar{Q}]$.

Lemma 3.1. Let $V$ be a Whitney structure and $\left\{L_{1}, \ldots L_{n-1}\right\}$ a local basis for $V$ near a point of $\Sigma$. Then $\operatorname{det}\left(\mathcal{L}\left(L_{j}, \overline{L_{k}}\right)\right)$ has only simple zeroes.

Remark 3.1. The example following the proof shows that the lemma does not hold if $V$ is only assumed to be almost Whitney.

Proof. It is sufficient to prove this for one basis and near one point $p \in \Sigma$. Let $\mathcal{O}$ be a neighborhood in $\mathcal{N}$ of $p$. Using an argument similar to that for Lemma 1.2, we see that there is a basis $\left\{X_{1}, \ldots, X_{n-1}, Y\right\}$ for $T M$ over $\mathcal{O}$ such that $\left\{X_{1}, \ldots, X_{n-1}\right\}$ is a basis for $V$ over $\mathcal{O} \cap \Sigma$ and $\left\{X_{1}+i B_{1} Y, \ldots, X_{n-1}+i B_{N-1} Y\right\}$ is a basis for $V$ over $\mathcal{O}$, where each $B_{j}$ is real and is zero on $\Sigma$. Set $L_{j}=X_{j}+i B_{j} Y$. Note that, as a consequence of involutivity, $\omega\left(\left[L_{j}, L_{k}\right]\right)=0$ and so, on $\Sigma$

$$
\begin{aligned}
\mathcal{L}\left(L_{j}, \overline{L_{k}}\right)=\omega\left[L_{j}, \overline{L_{k}}\right] & =\omega\left[L_{j}, L_{k}-2 i B_{k} Y\right] \\
& =-2 i \omega\left[L_{j}, B_{k} Y\right] \\
& =-2 i L_{j}\left(B_{k}\right) \omega(Y) \\
& =-2 i X_{j}\left(B_{k}\right) \omega(Y) .
\end{aligned}
$$

Since $\omega(Y) \neq 0$, we seek to prove that $\operatorname{det}\left(X_{j}\left(B_{k}\right)\right)$ has only simple zeroes. We have already used involutivity; now we use the conditions for an almost Whitney structure. First we rewrite this determinant as $d B(X)$, where $d B=d B_{1} \wedge \ldots \wedge d B_{n-1}$ and $X=X_{1} \wedge \ldots \wedge X_{n-1}$. Let $\left\{\theta_{1}, \ldots, \theta_{n}\right\}$ be the basis dual to $\left\{X_{1}, \ldots, X_{n-1}, Y\right\}$. So $\omega=\sum A_{j} \theta_{j}$ with $A_{k}+i B_{k} A_{n}=0$. We may assume $A_{n}=1$ and so

$$
\omega=-i \sum_{1}^{n-1} B_{k} \theta_{k}+\theta_{n} .
$$


Our map $\Phi: M \rightarrow P C T^{*} M$ is

$$
x \mapsto\left(x,\left[-i B_{1}, \ldots,-i B_{n-1}, 1\right]\right),
$$

and the condition for transversality is $d B \neq 0$.

Let $\tau$ be a nonzero section of $T \Sigma$. Note that $i(\tau) d B=0$ for the interior multiplication of a vector and a differential form.

Case $1 . \omega(\tau) \neq 0$ at $p$.

Since $\omega\left(X_{j}\right)=0$, we may assume that $\tau=Y+\sum t_{j} X_{j}$. Then since $i(\tau) d B=0$ while $d B \neq 0$, we see that $d B(X) \neq 0$.

Case 2. $\omega(\tau)=0$ at $p$.

We may assume $\tau=X_{1}+\sum t_{j} X_{j}+t Y$ with $t$ and each $t_{j}$ being zero at $p$. Note that $\omega(\tau)=t$ and so, by our assumptions, $\left.t\right|_{\Sigma}$ vanishes to first order at $p$. Note also that now $i(\tau) d B=0$ implies that $i\left(X_{1}\right) d B=0$ at $p$, and so $d B\left(Y, X_{2}, \ldots, X_{n-1}\right) \neq 0$. Thus we have

$$
\begin{aligned}
d B(X) & =d B\left(\tau \wedge X_{2} \wedge \ldots \wedge X_{n}\right)-t d B\left(Y \wedge X_{2} \wedge \ldots \wedge X_{n}\right) \\
& =-t d B\left(Y \wedge X_{2} \wedge \ldots \wedge X_{n}\right)
\end{aligned}
$$

and this implies $\left.d B(X)\right|_{\Sigma}$ vanishes to first order at $p$.

For the example, let $V$ have as a local basis

$$
\begin{array}{llc}
L_{1} & = & \partial_{y}-i y \partial_{x}, \\
L_{2} & = & \partial_{t}-\left(\sqrt{2} y+\frac{i}{2} t\right) \partial_{x} .
\end{array}
$$

$V$ is not Whitney since $\left[L_{1}, L_{2}\right] \notin V$. The map $\Phi: M \rightarrow P C T^{*} M$ is given by

$$
(x, y, t) \rightarrow\left(x, y, t, i y, \sqrt{2} y+\frac{i t}{2}\right)
$$

so $\Sigma$, which is the projection of $\Phi(M) \cap P T^{*} M$, is given by $\{(x, 0,0)\}$, and $\Phi(M)$ clearly is transverse to $P T^{*} M$. Further, $\omega\left(\partial_{\theta}\right)$ is never zero on $\Sigma$. Thus $V$ is an almost Whitney structure. But, as is easily seen, $\operatorname{det} \mathcal{L}\left(L_{j}, \overline{L_{k}}\right)$ is identically zero.

\section{A special case of the Bott Vanishing Theorem}

Bott has found necessary conditions for a real (or holomorphic) bundle to be isomorphic to the tangent bundle of a foliation. See for instance 2]. His method also applies to involutive sub-bundles of $C T M$. Here we present the simplest case of his calculations.

Theorem 4.1. Let $M=C P^{n}$, for some $n \geq 2$. There exists a co-rank one subbundle of CTM which is not isomorphic to any involutive sub-bundle of CTM.

Proof. Let $V$ be a co-rank one involutive sub-bundle and let $Q$ be any line bundle with

$$
V \oplus Q=C T M
$$

We follow 2 to show that $c_{1}(Q)=0$. It is sufficient to work in $H^{2}(M, R)$, and so we will use the curvature of a connection to get a two-form which represents this Chern class. Let

$$
\pi: C T M \rightarrow Q
$$

be the projection associated to the decomposition (4). Use a partition of unity to find a global connection which satisfies

$$
\nabla_{X} \zeta=\pi[X, \zeta]
$$


for $X \in V$ and $\zeta$ a local section of $Q$. For the curvature of the connection we have

$$
\begin{aligned}
R(A, B) \zeta & =\left(\nabla_{A} \nabla_{B}-\nabla_{B} \nabla_{A}-\nabla_{[A, B]}\right) \zeta \\
& =\omega(A, B) \zeta
\end{aligned}
$$

where $A$ and $B$ are in $T M$. The two-form $\omega$ represents $c_{1}(Q)$ as an element of $H^{2}(M, R)$. The Jacobi identity shows that $R(A, B) \zeta=0$ when $A$ and $B$ are both in $\Re V$. It follows that $\omega \wedge \omega=0$. Thus $c_{1}(Q)^{2}=0$, and this implies, since $H^{*}(M, R)$ is a truncated polynomial ring, that $c_{1}(Q)=0$. So when $V$ is involutive, $Q$ is trivial.

It is easy to see that any complex line bundle over $M$ embeds into $C T M$. (This holds for all manifolds.) So choose some nonzero $\alpha \in H^{2}(M, Z)$ and let $Q_{0}$ be some complex line in $C T M$ with first Chern class equal to $\alpha$. Thus no bundle $V_{0}$ with

$$
V_{0} \oplus Q_{0}=C T M
$$

can be involutive. Moreover, $V_{0}$ cannot even be isomorphic to an involutive subbundle. For, say $V_{0} \cong V$ with $V$ involutive. Then

$$
V_{0} \oplus Q_{0}=V \oplus Q
$$

implies that $c_{1}\left(Q_{0}\right)=c_{1}(Q)=0$.

\section{REFERENCES}

[1] M. Atiyah, Vector fields on manifolds, Arbeitsgemeinschaft für Forschung des Landes Nordrhein-Westfalen, Heft 200 Westdeutscher Verlag, Cologne 1970. MR0263102 (41:7707)

[2] R. Bott, Lectures on characteristic classes and foliations, Notes by Lawrence Conlon, with two appendices by J. Stasheff. Lectures on algebraic and differential topology (Second Latin American School in Math., Mexico City, 1971), pp. 1-94. Lecture Notes in Math., Vol. 279, Springer, Berlin, 1972. MR0362335 (50:14777)

[3] H. Jacobowitz, Whitney and Mizohata structures, Contemporary Mathematics, in Geometric analysis of PDE and several complex variables, 293-304, Contemp. Math., 368, Amer. Math. Soc., Providence, RI. MR.2126476 (2005m:58042)

[4] L Maps into complex space, Proc. Amer. Math. Soc. 134 (2006), no. 3, 893-895 (electronic). MR2180907

[5] H. Jacobowitz and G. Mendoza, Sub-bundles of the complexified tangent bundle, Transactions of the AMS 355 (2003), 4201-4222. MR1990583(2005a:58030)

[6] H. Levine, Elimination of cusps, Topology 3 (1965), suppl. 2, 263-296. MR0176484 (31:756)

[7] E. Thomas, Fields of tangent k-planes on manifolds, Inventiones Math. 3 (1967), 334-347. MR0217814(36:903)

[8] E. Thomas, Fields of tangent 2-planes on even-dimensional manifolds, Ann. of Math. (2) 86 (1967), 349-361. MR0212834(35:3699)

[9] H. Whitney, On the singularities of mappings of Euclidean spaces I, Mappings of the plane into the plane, Annals of Math. 62 (1955), 374-410. MR0073980 (17:518d)

Department of Mathematics, Rutgers University, Camden, New Jersey 08102

E-mail address: jacobowi@camden.rutgers.edu 